\title{
Perspectivas epistemológicas sobre el cambio conceptual: Implicaciones para la práctica educativa
}

\author{
Richard A. Duschl
}

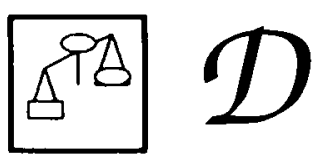

En este artículo se presenta un modelo gradual del carácter y el mecanismo de la reestructuración del conocimiento y, a continuación, se describe un modelo de práctica educativa (la evaluación por carpetas) diseñado para facilitar esta forma de reestructuración. Este modelo educativo crea oportunidades para que enseñantes y sobre todo estudiantes puedan confrontar y desarrollar su comprensión científica, puedan aumentar su responsabilidad por su propia reestructuración del conocimiento y puedan ellos mismos evaluar cuando se trata de enseñar ciencia.

La mayoría de nosotros estaría de acuerdo en que los estudiantes reestructuran el conocimiento. En la enseñanza de la ciencia, la cuestión de la reestructuración del conocimiento se encarna en los modelos de enseñanza de cambio conceptual (Anderson y Smith, 1986; Champagne, 1988; Osborne y Wittrock, 1983; Roth, 1989; Treagust y Fetherstonhaugh, 1990; Zeitsman y Clement, 1990 ), que aducen que para la implementación de estrategias educativas que pretenden enmendar las diferencias existentes entre las teorías y significados de conceptos científicos que tienen los niños, y las teorías y significados que los científicos tienen de los mismos conceptos. Un aspecto educativo destacado de las teorías de enseñanza-aprendizaje basadas en el cambio conceptual es determinar la mejor manera de introducirlas en las aulas para que puedan influir en la toma cotidiana de decisiones educativas.

Sin embargo, los marcos de referencia que reconocen los cambios que se producen en el conocimiento no son suficientes para trazar un curso educativo. La investigación también debe intentar comprender cómo se producen estos cambios en la reestructuración del conocimiento y cómo construir un entorno de aprendizaje que facilite esta reestructuración. Estas consideraciones plantean importantes interrogantes y cuestiones de carácter filosófico, psicológico y pedagógico sobre cómo se produce el cambio conceptual y qué características del crecimiento del conocimiento deberían formar parte de los currículos y de los entornos de aprendizaje. En el hecho de destacar el cómo, se encuentra implícito un cambio en la perspectiva de la enseñanza de la ciencia, que va desde abrazar «los modos de conocimiento de los científicos» como objetivo dominante a favorecer «el posicionamiento del estudiante para 
el próximo paso». Este cambio de perspectiva y de enfoque representa un desvío radical y complejo en relación a la práctica común.

El propósito de este artículo es articular y presentar una explicación del carácter y el mecanismo de la reestructuración y, a continuación, describir un modelo de práctica educativa diseñado para facilitar esta forma de reestructuración. En este modelo educativo ocupa un lugar central un punto de vista ampliado e integrado de la evaluación y la enseñanza que nosotros denominamos cultura de la carpeta. La característica esencial de esta cultura es que crea oportunidades para que enseñantes y estudiantes puedan confrontar y desarrollar su comprensión científica, y que permite equipar a los estudiantes con las herramientas necesarias para aumentar su responsabilidad por su propia reestructuración y para evaluar por sí mismos cuáles pueden ser los siguientes pasos.

El papel de la evaluación en esta empresa es fundamental, pero también es totalmente diferente del papel que ha desempeñado tradicionalmente. El proceso de evaluación debería caracterizarse por realizar, evaluar, discutir y poner en duda afirmaciones relativas al conocimiento. En realidad, la evaluación misma debería ser un producto conjunto de enseñante y estudiante que, en gran medida, recapitule exámenes científicos de fenómenos. Una evaluación cuidadosa de las declaraciones de conocimiento de los estudiantes puede ayudar a los enseñantes a diseñar experiencias educativas que fuercen una confrontación con estas creencias y que, por tanto, estimulen una reestructuración conceptual. Es de extrema importancia que los estudiantes aprendan a abordar los procesos de evaluar declaraciones de conocimiento, en vez de limitarse a aprender teorías cada vez más útiles.

La evaluación es fundamental para la enseñanza del cambio conceptual al menos por tres razones. En primer lugar, la comprensión que los estudiantes aportan a la situación de aprendizaje es crucial. En segundo lugar, la enseñanza del cambio conceptual está muy orientada hacia la comprensión de los procesos de crecimiento conceptual y no solo de los resultados, procesos que se pueden clarificar mediante técnicas de evaluación adecuadas. En tercer lugar, la evaluación siempre ha desempeñado un papel importante en la comunicación de los criterios de rendimiento que se valoran dentro de una disciplina. Por desgracia, con frecuencia lo que se ha comunicado ha sido insatisfactorio en términos de su relación con la disciplina científica. Si se construye adecuadamente, la evaluación puede ayudar a articular, para estudiantes y enseñantes, cualidades y valores importantes para la empresa científica.

Empezamos con una discusión de cómo las epistemologías de la ciencia sirven para fundamentar la práctica orientada a la reestructuración del conocimiento. La postura adoptada aquí es que los investigaciones que examinan aspectos de la reestructuración del conocimiento en la actualidad, están empleando modelos epistemológicos que no dan cabida al pensamiento contemporáneo de los filósofos de la ciencia; tampoco ofrecen una descripción satisfactoria de los mecanismos de la reestructuración del conocimiento. Se encuentra en juego la sensación que tiene el estudiante de la racionalidad de la ciencia y el reconocimiento de que la reestructuración es fundamental para el desarrollo científico.

Por tanto, en el próximo apartado del artículo se revisan y comentan los fundamentos epistemológicos de la investigación sobre el cambio conceptual. Revisamos los primeros trabajos espoleados por Kuhn (1962) y Lakatos (1970) y luego examinamos cómo otros desarrollos más recientes en la filosofía de la ciencia que abogan por una concepción gradual y semántica del crecimiento de las teorías científicas, pueden impulsar a las nociones actuales de la enseñanza del cambio conceptual. A continuación revisamos investigaciones de la ciencia cognitiva y de la práctica educativa que examinan mecanismos que apoyan al cam- 
bio conceptual. En el último apartado presentamos un modelo educativo de una cultura de carpeta que tiene el potencial para of recer un contexto dentro del cual se puede llevar a cabo la enseñanza de esta forma de cambio conceptual.

\section{FUNDAMENTOS DEL CAMBIO CONCEPTUAL}

\section{Puntos de vista iniciales}

Desarrollos contemporáneos en los campos académicos de la historia y la filosofía de la ciencia, y de la ciencia cognitiva, han producido resultados que sugieren que los procedimientos para describir y analizar el crecimiento del conocimiento, tanto en un campo de estudio (desarrollo de teorías) como en los individuos (teorías de esquemas), tienen mucho en común y se pueden describir mediante un lenguaje común (Duschl, Hamilton y Grandy, 1990; véase también Carey, 1985a, 1985b; Chi, 1990; Rissland, 1985; Vosniadou y Brewer, 1987). El proceso de desarrollo de teorías por parte de los científicos con frecuencia ha sido comparado al desarrollo y la adquisición de conocimientos sobre el mundo por parte de un individuo (Kitchener, 1986, 1987, en prensa; Krupa, Selman y Jaquette, 1985; Piaget, 1970). Los marcos de referencia que emplean términos comunes —esquemas, marcos esquemáticos, teoría, cambio conceptual - abordan las actividades asociadas al crecimiento del conocimiento o su reestructuración, es decir, a la reestructuración débil o radical de las teorías de los estudiantes. Cada ámbito de investigación, tanto si se centra en los individuos como si se centra en las teorias, debe determinar qué cosas cuentan como evidencia prototípica del crecimiento del conocimiento (ejemplares) y como evidencia contraria (datos anómalos). Por tanto, cabe dar la bienvenida a la petición de Carey (1986) de que los enseñantes de ciencias consideren las contribuciones que pueden hacer los principios y los conceptos de la historia y la filosofía de la ciencia a los investigadores que intentan interpretar y comprender el desarrollo conceptual de estudiantes jóvenes.

Una manera efectiva de concebir lo que interviene en el proceso de reestructuración del cambio conceptual es considerar la noción de Hanson de observar o "ver". En su clásica obra Patterns of Discovery (1958), Hanson distingue entre "ver como", que es una observación que se produce sin el beneficio de un conocimiento de fondo apropiado, y "ver que", que implica observaciones con un trasfondo adecuado de conocimientos. En cierto sentido, la tarea a la que se enfrentan los enseñantes de ciencias es tomar a individuos que «ven como» y ayudarles a llegar a ser observadores que «vean que». Se trata de una versión simplista, aunque también exacta, de la enseñanza y el aprendizaje del cambio conceptual.

Algunos modelos del cambio conceptual, como el modelo propuesto por Posner, Strike, Hewson y Gertzog $(1982)^{1}$, están basados en los argumentos epistemológicos de Kuhn (1962) y Lakatos (1970), según los cuales el crecimiento del conocimiento científico se desarrolla mediante períodos cíclicos de consenso (los episodios de ciencia normal de Kuhn) y disensión (episodios de ciencia revolucionaria) entre los practicantes. En realidad, estos puntos de vista han conducido a una perspectiva en la investigación del aprendizaje de la ciencia que categoriza el cambio del conocimiento en dos tipos fundamentales: reestructuración débil y reestructuración radical (por ejemplo, Carey, 1985a; Chi, 1990; Vosniadou y Brewer, 1987). La reestructuración débil procede de la idea de Kuhn de alteraciones durante la "ciencia normal» que funcionan dentro de los mismos ejemplares ${ }^{2}$ y de la idea de Lakatos de cambios en el «núcleo blando» de un programa de investigación. En este caso, el marco esquemático del estudiante es aceptable. En cambio, la reestructuración fuerte es similar a la "ciencia revolucionaria» de Kuhn, en la que se adoptan nuevos ejempla- 


\section{0}

res, y a la idea de Lakatos del abandono del «núcleo duro» de compromisos teóricos de un programa de investigación. En este caso, el marco esquemático del estudiante no es aceptable y debe ser sustituido.

Posner $e t$ al. sugieren que este punto de vista del cambio conceptual sobre el crecimiento del conocimiento científico se puede aplicar a la enseñanza. Sugieren que, para que los estudiantes modifiquen sus compromios con ideas o marcos de referencia esquemáticos científicos, se deben cumplir cuatro condiciones.

1. Las ideas existentes deben verse como insatisfactorias.

2. La idea nueva debe ser inteligible, coherente e internamente consistente.

3. La idea nueva debe ser plausible.

4. La idea nueva debe ser preferible al antiguo punto de vista en base a la elegancia, la simplicidad y/o la utilidad percibidas.

Sin embargo, los modelos epistemológicos de Kuhn y Lakatos que se utilizan para guiar el pensamiento educativo son problemáticos. Los niños son muy reticentes a cambiar sus conceptos incluso ante una enseñanza que busca este cambio. Una reciente revisión de la investigación sobre el aprendizaje de la ciencia realizada por Gunstone, White y Fensham (1988), lamenta que los intentos realizados para modificar las concepciones de los estudiantes suelan carecer de éxito y consuman mucho tiempo.

Quizá la limitación fundamental para la aplicación de la teoría del cambio conceptual de Posner $e t$ al. al aprendizaje de la ciencia (y de las diversas versiones kuhnianas y lakatusianas empleadas por los científicos cognitivos citados anteriormente) es que se basa en una epistemología que adopta un modelo jerárquico de la justificación del conocimiento. Un modelo del cambio conceptual es jerárquico cuando presupone que los cambios en los compromisos fundamentales de una teoría de la ciencia producen cambios simultáneos en otros compromisos ontológicos, metodológicos y axiológicos dentro del marco conceptual. Estudios de la historia de la ciencia han documentado que la ciencia progresa a través de períodos tanto de consenso como de disensión entre sus practicantes, y que estos cambios no implican cambios holísticos globales sino que presentan un carácter más bien gradual (Laudan, 1984; Nersessian, 1989; Shapere, 1984; Thagard, 1990). Lo que está en juego es la caracterización del cambio científico como proceso holístico. Más concretamente, el objeto de la discusión es la representación holística de que el cambio en los marcos conceptuales es simultáneo en vez de secuencial.

Esencialmente, un modelo jerárquico de cambio conceptual da un énfasis indebido al estado ontológico de los esquemas conceptuales, en detrimento de las consideraciones en torno a cómo influyen los compromisos metodológicos y axiológicos en estos esquemas conceptuales. Según Laudan (1984), este punto de vista holístico de los cambios en la teoría científica es erróneo por varias razones. En primer lugar, describe de manera inexacta cómo se producen el consenso y la disensión en la ciencia. En segundo lugar, destaca el papel del consenso en la ciencia por encima de la disensión. Por último, pasa por alto los importantes papeles desempeñados por los objetivos cognitivos y la metodología cognitiva en el establecimiento de la evidencia para producir el cambio conceptual.

Existen problemas con la noción de compromiso central y con las condiciones necesarias para provocar un cambio conceptual, tal como las definieron originalmente Kuhn y Lakatos, cuando se aplican al desarrollo de un mecanismo para describir cambios entre consenso y disensión. Específicamente, las concepciones sufren de la «falacia de la covarianza» (Laudan, 1984, p. 43) ya que se presupone que la medida en que los científicos (o, como se aduce posteriormente, los enseñantes y los estudiantes) se ponen de acuerdo respecto a los compromisos centrales de un nivel, determina la medida del acuerdo en todos los niveles restantes. Los niveles de interés son 
(a) afirmaciones o teorías objetivas, (b) métodos y (c) objetivos o fines que operan en un ámbito de la ciencia. Esta falacia refleja la tendencia a presuponer que unas divergencias importantes en las creencias sobre teorías también acaban por provocar diferencias en los niveles de los fines, los objetivos y los métodos.

\section{Puntos de vista actuales}

Un punto de vista del cambio científico alternativo al punto de vista holístico es la perspectiva gradual del cambio científico de Laudan (1984). La «matriz disciplinar» de la ciencia de Kuhn (1970), que se encuentra en el comentario final a la segunda edición de The Structure of Scientific Evolution, también reconoce este punto de vista. El enfoque gradual de Kuhn describe la ciencia como un proceso que funciona dentro de una matriz disciplinar (véase la Figura 1). La matriz disciplinar está formada por cuatro tipos de componentes: (a) componentes lógicos formales, (b) creencias en modelos particulares, incluyendo modelos de variedad heurística - moléculas de gas como bolas de billar-, (c) valores y (d) ejemplares. En el comentario final de Kuhn encontramos una perspectiva según la cual los cambios en las concepciones se producen mediante cambios en las partes y no en el todo. La matriz disciplinar alude a la naturaleza compleja de las teorías. Kuhn escribe que «sin embargo, tal como se utiliza actualmente en filosofía de la ciencia, "teoría" implica una estructura mucho más limitada en cuanto a naturaleza y alcance que la requerida aquí»" (Kuhn, 970, p. 182). Así pues, de esto se desprende que la matriz disciplinar también alude a la naturaleza compleja de la reestructuración del conocimiento. Por desgracia, la discusión que hace Kuhn de este importante tema es muy limitada.

Laudan (1984) ofrece un análisis más amplio de la naturaleza de la reestructuración que Kuhn. Para Laudan, la noción de cambio se caracteriza porque los cambios en la ontología, la metodología y la axilogía pueden darse, y se dan, por separado y dentro de períodos de tiempo distintos y mutuamente excluyentes:

Donde la imagen reticulada difiere de manera más fundamental de la jerárquica, es en la insistencia de que existe un proceso complejo de ajuste y justificación mutua entre los tres niveles del compromiso científico. La justificación fluye hacia arriba y hacia abajo en la jerarquía, vinculando objetivos, métodos y afirmaciones objetivas. Ya no debemos considerar a ninguno de estos niveles como privilegiado o primario o como más fundamental que los restantes. La axiología, la metodología y las afirmaciones objetivas están inevitablemente entrelazadas en relaciones de dependencia mutua. El «orden de picoteo» implícito en el enfoque jerárquico debe ceder el paso a un tipo de principio de nivelación que destaca las pautas de dependencia mutua entre estos diversos niveles. (Laudan, 1984, p. 62).

Figura 1

Matriz disciplinar de Kubn para la reestructuración del conocimiento

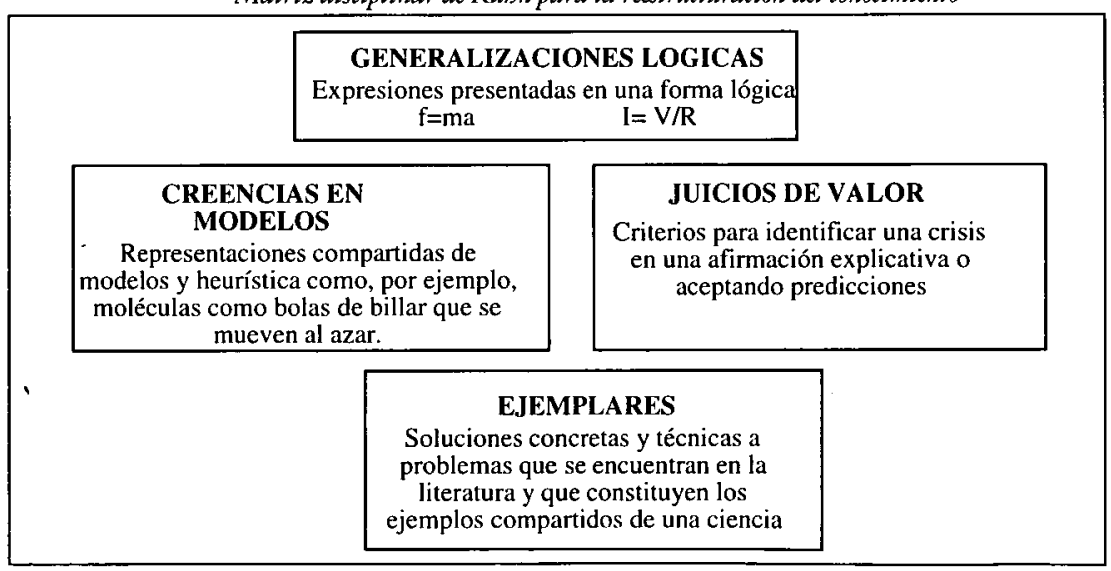


Entre los ejemplos que apoyan el punto de vista de Laudan cabe incluir la investigación de Whitr $(1989,1990)$ sobre la búsqueda de teorías llevada a cabo por los químicos del siglo XIX, donde describe cómo los científicos realizaban investigaciones sobre el modelo atómico de Dalton sin creer en él. También el trabajo de Bechtel (1990) muestra el papel de la instrumentación experimental para establecer evidencias científicas en el campo de la investigación biomédica.

Laudan propone lo que él denomina «modelo reticulado» (véase la Figura 2) y aduce que la justificación del conocimiento científico implica un componente evolutivo que se puede representar mediante una red triádica en la que:

1. Los fines justifican la metodología y deben armonizar con la teoría.

2. La metodología justifica la teoría y cumple con los objetivos.

3. La teoría constriñe a la metodología y armoniza con los objetivos.

FIGURA 2

La Red Triádica de la Justificación (de Laudan, 1984, p. 63)

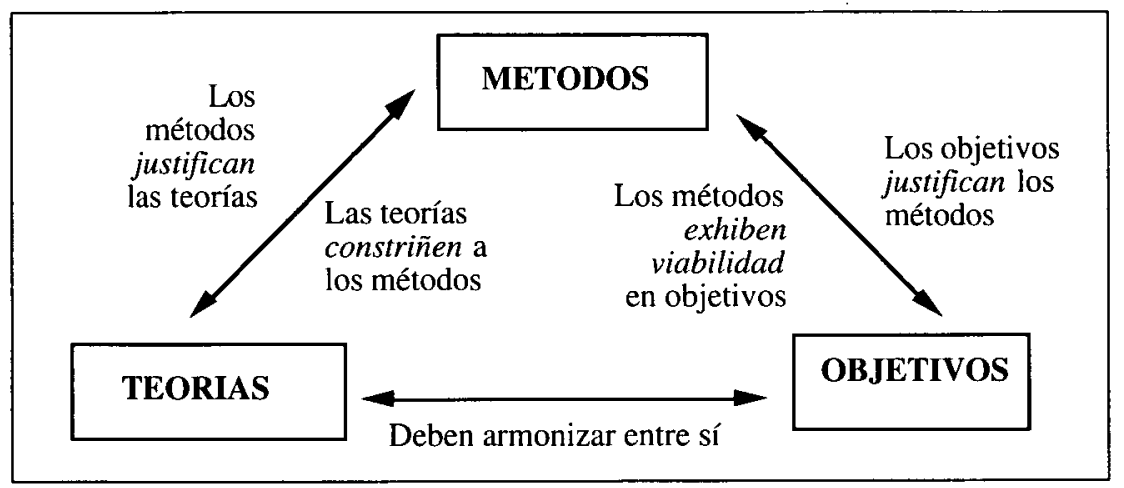

Según Laudan, las ventajas de la red triádica es que abandona los modelos jerárquicos del cambio según los cuales un cambio en la teoría acarrea automáticamente cambios en la metodología y en los fines. El modelo de la red triádica ofrece la posibilidad de que los científicos que trabajan en una disciplina puedan alterar sus compromisos teóricos y, al mismo tiempo, seguir manteniendo compromisos metodológicos y axiológicos desarrollados a partir de un marco teórico previo.

Con todo, la enseñanza de la ciencia suele ser culpable de presentar el crecimiento del conocimiento científico en un marco holístico. Nuestros currículos suelen presentar imágenes del cambio con unas pinceladas tan amplias que enmascaran el marco temporal y los sucesos cruciales que provocan los cambios, tanto en lo que sabemos como en la manera en que hemos llegado a saberlo. La aplicación de la red triádica como una epistemología para guiar la toma de decisones educativas debería ayudar a los educadores a poner en duda el "principio de nivelado" que está integrado en nuestra enseñanza de la ciencia, en nuestra planificación de lecciones y en nuestros programas de investigación. El marco epistemológico de Laudan nos recuerda la necesidad de incluir los cambios igualmente importantes de la metodología y los fines. Cuando miramos las partes, estamos mejor informados sobre cómo presentar de una manera significativa la ciencia a estudiantes no iniciados y ofrecerles estrategias para construir o reconstruir su conocimiento científico.

Por ejemplo, consideremos una unidad educativa sobre la tectónica de placas en una clase de ciencias terrestres. Un enfoque basado en la lógica del desarrollo que utilice un modelo epistemológico gradual para el estudio de la tectónica de placas, abordaría el importante papel desempeñado por los físicos británicos en el resurgir de la idea de la deriva continental. Inicialmente, el objetivo de estos científicos fue 
obtener evidencias sobre su teoría de la dínamo para explicar el campo magnético terrestre. Para tal fin se construyó un instrumento denominado magnetómetro astático. Estos científicos no encontraron evidencias para su teoría magnética pero sí descubrieron que el instrumento diseñado para comprobar teorías sobre el núcleo magnético de la Tierra también se podía utilizar para determinar el magnetismo residual de las rocas.

El descubrimiento de que el magnetómetro astático se podía utilizar para documentar el paleomagnetismo de las rocas, constituyó un paso crucial en el compromiso con el método para el avance del conocimiento geofísico de la tierra. Estudios posteriores de rocas continentales de Inglaterra, la India y Norteamérica produjeron un conjunto de datos anómalos que exigían una reestructuración del pensamiento dominante sobre la dinámica geológica. Al utilizar un punto de vista caracterizado por un contexto de desarrollo, encontramos que un cambio de método (utilizar el magnetómetro astático para medir rocas) condujo a un cambio en el objetivo cognitivo (los físicos apartaron sus recursos y sus esfuerzos de un problema clásico de magnetismo y los redirigieron hacia el problema de la deriva continental) que, a su vez, condujo a una reestructuración total de nuestro conocimiento sobre la Tierra. (Véase en Takeuchi, Uyeda y Kanamori, 1967, una narración completa de este episodio del descubrimiento científico).

\section{REESTRUCTURACIÓN DE LOS CONCEPTOS DE LOS ESTUDIANTES}

La adopción de la postura de que los entornos de aprendizaje deberían facilitar las formas de cambio conceptual observadas en la ciencia, implica que necesitamos comprender los mecanismos mediante los cuales los científicos realmente pasan de un período de consenso a otro de disensión o viceversa. Es por esta razón, entre otras, que los filósofos de la ciencia se han interesado fervientemente por el desarrollo y la reestructuración de las declaraciones de conocimiento científico. Los problemas esenciales de la filosofía de la ciencia han sido los que examinan la estructura de las teorias científicas (Suppe, 1977, 1989), porque se cree que las teorías encarnan el cambio conceptual.

Nuestras concepciones de entornos de aprendizaje para el cambio conceptual también se han visto afectadas por avances en el campo de la psicología cognitiva. Los estudiantes son percibidos como agentes activos en el proceso de construir significados (Brown, Collins y Duguid, 1989; Cognition and Technology Group at Vanderbilt, 1990; Resnick y Klopfer, 1989). La enseñanza constructivista está caracterizada por una reestructuración, una modificación y una adaptación continuas de declaraciones de conocimiento, además de métodos y objetivos de investigación. Entre los ejemplos de nuevos enfoques de la ciencia que utilizan marcos de aprendizaje y de materias que destacan la reestructuración del conocimiento, cabe incluir los siguientes:

1. El ciclo de aprendizaje ${ }^{3}$ (Champagne, 1988).

2. El modelo de enseñanza del cambio conceptual (Anderson y Smith, 1986; Minstrell, 1989; Roth, 1989; Roth, Anderson y Smith, 1987; West y Pines, 1985).

3. El modelo de aprendizaje generativo (Osborne y Wittrock, 1983; Osborne y Freyberg, 1985).

4. El empleo de analogías y de puentes analógicos (Duit, 1990; Zeitsman y Clement, 1990).

En un entorno de enseñanza del cambio conceptual, el currícúlo y el entorno de aprendizaje están diseñados para fomentar la construcción de teorías respe- 
tando, al mismo tiempo, los sistemas de creencias que poseen los estudiantes en cada momento. Con frecuencia, estas teorías de los estudiantes recapitulan el desarrollo histórico del pensamiento científico (por ejemplo, Nersessian, 1989; Nussbaum, 1983; Thagard, 1990). La evaluación puede proporcionar la oportúnidad para que las concepciones actuales de los estudiantes sean confrontadas y desafiadas y para que, mediante un conjunto de interacciones guiadas por el enseñante, se reestructuren teorías. Linn (1986), Novak (1977), Novak y Gowin (1984), Resnick (1983), Finley (1983), Anderson y Smith (1986) y Krupa et al. (1985), entre otros, hablan del efecto del conocimiento anterior de un estudiante en su aprendizaje posterior. El conjunto colectivo de estas investigaciones implica que los estudiantes, como asevera Carey (1986), desarrollan sus aptitudes cognitivas mediante el proceso de modificar progresivamente esquemas conceptuales.

Los argumentos en pro de que las estructuras de las teorías científicas y los esquemas cognitivos tienen mucho en común (Carey, 1986; Gibson, 1985; Giere, 1988), deben tener en cuenta tanto las características estructurales como evolutivas de estos marcos conceptuales. Desde una perspectiva estructural, las teorías científicas y los esquemas cognitivos realmente pueden tener mucho en común. Las teorías se pueden considerar como compuestas por hechos y principios, además de afirmaciones legítimas que son moldeadas conjuntamente mediante prácticas metodológicas y axiológicas aceptadas. Los esquemas cognitivos también se pueden concebir como formados por conceptos y afirmaciones proposicionales gobernados por reglas y valores que guían la síntesis.

Un aspecto fundamental de la aplicación de la epistemología a la enseñanza de la ciencia comporta describir los mecanismos para el cambio en la estructura de teorías o de esquemas conceptuales y su relación con el crecimiento del conocimiento. Además, la investigación de la dinámica del cambio conceptual ha añadido otro elemento importante a la tarea: El marco epistemológico del estudiante. Strike y Posner (en prensa), Schauble y Klopfer (1990), Kuhn, Amsel y O'Loughlin (1988) y Carey et al. (1989) han obtenido evidencias que sugieren que el marco epistemológico de un estudiante es un factor que contribuye a efectuar cambios en la representación del conocimiento. En el nivel de las aulas, se traduce en lo que los estudiantes consideran que es evidencia a favor o en contra de una explicación científica emergente. ¿Cómo se cuentan como evidencia los datos ejemplares y los datos anómalos en la reestructuración del conocimiento? ¿Cómo se puede utilizar la evaluación para ayudar a los estudiantes a compartir los criterios que gobiernan los juicios sobre la calidad de las afirmaciones y las evidencias de la ciencia?

Las perspectivas actuales sobre las epistemologías de los estudiantes se basan principalmente en puntos de vista filosóficos de la reestructuración de teorías. De éstos, los más destacados son los puntos de vista de Kuhn y de Lakatos quienes, junto con otros filósofos, participaron en el desmantelamiento del positivismo lógico y lo sustituyeron por la «nueva filosofía de la ciencia» (Brown, 1977). Este nuevo punto de vista adopta la idea de que los conceptos, las explicaciones y los procesos científicos están imbuidos de teoría, es decir, no son independientes de anteriores compromisos de conocimiento. Por tanto, recordando a Hanson, lo que cuenta está influido por el conocimiento que elegimos adoptar. En la medida en que el conocimiento que adoptamos cambie con el tiempo, lo que llegamos a considerar o a contar como evidencia a favor o en contra de una afirmación también sufre una reestructuración; este punto de vista que ha sido adoptado sumariamente por investigadores de la educación (Anderson y Smith, 1986; Cobb, Wood y Yackel, 1991; Linn, 1986; Posner et al., 1982) y por cientí- 
ficos cognitivos (Carey, 1986; Carey et al., 1989; Chi, 1990; Kuhn, Amshel y O'Loughlin, 1988; Vosniadou y Brewer, 1987).

Se ha demostrado que el pensamiento científico está enraizado en los detalles particulares de un ámbito (Glaser, 1984). Por tanto, se debe enseñar el conocimiento declarativo o específico de un dominio, relacionándolo con principios, leyes, teorías y generalizaciones de la ciencia, junto con los conocimientos estratégicos procedimentales/genéricos del dominio. Dentro del contexto de los desarrollos científicos normales o de la reestructuración débil, existe una pequeña cantidad de conocimiento procedimental que se debe adquirir sobre el ajuste fino de teorías y el ajuste de relaciones conceptuales. Pero si deseamos producir una reestructuración radical de conceptos, el equivalente personal de la ciencia revolucionaria de Kuhn, entonces parece que también debemos enseñar el conocimiento procedimental implicado en la evaluación de teorías, evidencias, observaciones y datos. Los principios epistemológicos contemporáneos aluden a la importancia de establecer una base para aceptar teorías científicas como si fueran a la vez revisionistas y provisionales (Suppe, 1977, 1989; Whitt, 1990). Por tanto, aunque es importante comprender cómo se generan las teorías, es igualmente importante comprender cómo se revisan, se alteran o se sustituyen. En pocas palabras, debemos estar preparados para participar en una enseñanza que fomente cambios en las epistemologías de los estudiantes, como sugieren Carey et al. (1989) y Strike y Posner (en prensa). Sin embargo, esta tarea no es sencilla. Duschl, Hamilton y Grandy (1990) sugieren que la naturaleza del conocimiento procedimental ha sido poco estudiada y en modo alguno se ha llegado a un acuerdo sobre ella.

Es muy posible que la naturaleza evasiva de la enseñanza del cambio conceptual (Gunstone $e t$ al., 1988) se deba a una infravaloración de la dinámica del currículo, la enseñanza y la gestión del aula en entornos educativos orientados a la comprensión. Las investigaciones de Tobin y Gallagher (1987), Duschl y Wright (1989) y Mitman, Mergendoller, Marchman y Packer (1987) apoyan esta opinión. Sin embargo, las recomendaciones de reforma que sugieren que el currículo de ciencias debería considerar la inclusión de temas que fomenten la construcción de significados en los estudiantes, aún tienen que explorar plenamente los procedimientos específicos para implementar un currículo de este tipo. Nuevos modelos epistemológicos que articulen detalles sobre el desarrollo de teorías pueden actuar como guía.

\section{LA CULTURA DE LA CARPETA: UN MODELO EDUCATTIVO DEL CAMBIO CONCEPTUAL}

Proponemos un modelo de la enseñanza de la ciencia denominado «cultura de carpeta" que tiene el potencial de abordar los aspectos planteados en esta discusión. Una cultura de carpeta es un medio de organizar y expresar el currículo y la enseñanza con el fin de fomentar interacciones en torno a una colección de trabajos: la carpeta. La etiqueta cultura pretende transmitir una imagen de un entorno de aprendizaje en clase que refleja una interacción extensiva entre enseñantes, estudiantes y currículo. Dos características básicas y distintivas del aula con cultura de carpeta son las interacciones basadas en evaluaciones entre enseñantes y estudiantes para supervisar el aprendizaje significativo, y la orientación hacia proyectos de las actividades y las tareas educativas. Las características que imaginamos para las aulas con cultura de carpeta en comparación con las aulas tradicionales se presentan en la Tabla I. 


\section{6}

TABLA I

Contraste entre las culturas tradicional y de carpeta en una clase de ciencias

\begin{tabular}{|c|c|}
\hline Cultura tradicional & Cultura de carpeta \\
\hline \multicolumn{2}{|c|}{ Punto de vista de la ciencia } \\
\hline Método cientifico hipotético-deductivo estricto & Método científico parcial \\
\hline Epistemología del Positivismo Lógico & Epistemología de realismo cientifico/concepción semántica \\
\hline Distinción sostenible entre observación y teoría & Distinción insostenible entre observación y teoria \\
\hline \multicolumn{2}{|c|}{ Papel del escudiante } \\
\hline Input bajo del estudiante/ imagen no activa & Input elevado del estudiantel imagen activa \\
\hline Significados científicos recibidos & Significados científicos negociados \\
\hline Nivel de reflexión bajo & Nivel de reflexión elevado \\
\hline Utilización de estrategias desarrolladas por el estudiante & Utilización de un conocimiento estratégico/ basado en principios \\
\hline \multicolumn{2}{|c|}{ Papel del enseñante } \\
\hline Difusor de conocimiento científico & Elabora el conocimiento científico \\
\hline No participa en la construcción de conocimiento científico & Participa en la construcción de conocimiento sobre la ciencia \\
\hline Adherencia estricta al currículo prescrito & Modifica y adapta el currículo prescrito \\
\hline \multicolumn{2}{|c|}{ Objetivos del currículo } \\
\hline Conocimientocientifico: Lo que sabemos & Conocimiento sobre la ciencia: Cómo y por qué sabemos \\
\hline $\begin{array}{l}\text { Destaca las explicaciones con una forma final totalmente } \\
\text { desarrollada }\end{array}$ & $\begin{array}{l}\text { Descaca el crecimiento del conocimiento y el desarrollo } \\
\text { de explicaciones }\end{array}$ \\
\hline Amplitud del conocimiento & Profundidad del conocimiento \\
\hline Conocimiento científico básico & Conocimiento científico contextualizado \\
\hline Unidades curriculares discretas & Unidades curriculares conectadas \\
\hline
\end{tabular}

Nuestra postura es que el modelo de la cultura de la carpeta debe adoptar un punto de vista de la enseñanza y el aprendizaje basado en el cambio conceptual. Pero también requiere un compromiso con un conjunto más elaborado de criterios epistemológicos, necesarios para capturar los elementos complejos e importantes de la reestructuración de conocimiento específica de cada ámbito. El trabajo de los estudiantes debería derivarse de un punto de vista epistemológico de la estructura de un ámbito. Las tareas y actividades educativas, y los problemas de la evaluación, no solo requieren las aptitudes que se consideren importantes para un ámbito, sino que estas aptitudes deberían integrarse en contextos que reflejaran una porción significativa de la estructura epistemológica.

Contemplar la enseñanza de la ciencia desde un punto de vista constructivista de «transformación del conocimiento», comporta que se deban tener en cuenta muchas cosas más en la composición del currículo que elegimos diseñar a implementar. Aunque una discusión completa de la cultura de la carpeta no es posible en este artículo, en los apartados que siguen destacaremos algunos aspectos cruciales de este currículo. Así pues, en el resto de este artículo nos centraremos en una vista general del modelo de educación basado en la cultura de la carpeta. El tema principal es un análisis de la evaluación. Entre los aspectos a considerar se incluye una discusión de los procesos y actividades educativos que se podrían utilizar en una aula con cultura de carpeta, discusión que va seguida de un examen de la dinámica de los criterios de evaluación.

\section{EVALUACIÓN}

El empleo de carpetas es un mecanismo que permite evaluar el conocimiento científico de los estudiantes. En una clase que utiliza carpetas, la evaluación se considera una actividad formativa, educativa y colaboradora que se produce entre enseñante y estudiante. La evaluación empieza haciendo públicos los criterios en base a los cuales se van a considerar los trabajos de los estudiantes. Estos criterios, en un sentido muy real, articulan los valores de un ámbito. La evaluación actúa como un 
vehículo sin igual para articular los objerivos de un estudio en un ámbito para todos los interesados en la empresa educativa. La evaluación es una actividad pública que es compartida por los estudiantes, los enseñantes y la institución educativa.

Las carpetas son, en parte, colecciones de trabajos de estudiantes que tienen las siguientes características. En primer lugar, estos trabajos emanan de actividades educativas que son experiencias científicamente válidas. Creemos que las actividades educativas deben concentrarse en los principios fundamentales que subyacen a un ámbito (por ejemplo, evaluar las declaraciones de conocimiento) en vez de concentrarse en los numerosos hechos y procedimientos que han constituido el currículo para la mayoría de los estudiantes. En segundo lugar, la carpeta necesita incluir evidencias del proceso de desarrollo conceptual del estudiante. Incluir únicamente informes finales, demostraciones y exámenes no ayudará, ni a estudiantes ni a enseñantes, a dilucidar el proceso de cambio conceptual. En tercer lugar, deben darse actividades reflexivas en torno a la carpeta. Es decir, los estudiantes necesitan aprender a pensar acerca de su trabajo y de su desarrollo conceptual de una manera científicamente legítima.

Las carpetas también se pueden utilizar para ayudar a los estudiantes a desarrollar una comprensión del crecimiento del conocimiento en la ciencia. La evaluación es un mecanismo mediante el cual los enseñantes y los estudiantes pueden participar en un discurso sobre la empresa científica. Este discurso tiene un significado especial porque está basado en las actividades de clase de estudiantes y enseñantes. Mediante un cuidadoso examen del proceso, y no solo de los resultados, los enseñantes tienen evidencias del éxito de distintos aspectos del currículo en fomentar los objetivos educativos.

Consideremos las inquietudes del enseñante en clase. Cuando presenta una enseñanza que intenta modelar, y al mismo tiempo provocar, cambios de conocimiento, es difícil demostrar que el crecimiento del conocimiento en la ciencia, con sus períodos de consenso y disensión es, de todos modos, una actividad racional. El problema de destacar los compromisos con la teoría $T$ y luego con las teorías $T 1, T 2$, etc. sin ninguna discusión de los métodos $M$, los objetivos $O$, o los marcos temporales, es que refuerza una versión de la ciencia acabada o final. Sin embargo, presentar el crecimiento del conocimiento científico únicamente como una serie de cambios teóricos, aumenta aún más el riesgo de representar el crecimiento del conocimiento científico como una serie de cambios irracionales y caprichosos entre científicos. Se dejan de lado los marcos temporales y los detalles de los sucesos y las evidencias perfectamente razonables y racionales que contribuyeron a los cambios en las creencias.

Sin embargo, la construcción y la evaluación de carpetas puede proporcionar a los estudiantes un modelo de cómo se producen estos cambios dentro de su propio pensamiento. En la carpeta no solo se deberían incluir evidencias de teorías, métodos y fines, sino también evaluaciones reflectivas que pueden ayudar a enseñantes y estudiantes a comprender el crecimiento del conocimiento en cada individuo. El lenguaje de evaluación concebido para caracterizar el desarrollo del conocimiento refleja los valores y las perspectivas epistemológicas que deseamos compartir con los estudiantes. En realidad, incluso podríamos imaginar a los estudiantes utilizando sus propias carpetas para examinar si el crecimiento de su conocimiento está mejor caracterizado como holístico o como gradual.

Hay tres implicaciones para la enseñanza que son coherentes con este punto de vista. En primer lugar, los objetivos curriculares y los planes para las lecciones deben centrarse en la comprensión que tienen los estudiantes de las explicaciones científ1cas, haciendo intervenir así la evaluación de evidencias, las declaraciones de conocimiento y los datos. En segundo lugar, es necesario desarrollar actividades educativas que estimulen la reestructuración de explicaciones. Por último, el trabajo de los 
estudiantes se deberá considerar o evaluar en términos coherentes con este punto de vista. Se ha visto que las prácticas educativas que se basan en una cultura de carpeta, como es el caso de Arts PROPEL (Gitomer, Grosh y Price, en prensa) y Lampert (1990), cumplen con eficacia estos objetivos.

\section{Procesos educativos}

Como se ha mencionado anteriormente, dos características básicas y distintivas de la clase con cultura de carpeta son las interacciones basadas en evaluaciones que mantienen los enseñantes con los estudiantes para supervisar la presencia de un aprendizaje significativo, y la orientación hacia proyectos de las actividades y las tareas educativas. Las prácticas educativas pertinentes incluyen interacciones basadas en evaluaciones que se producen en torno a una recopilación del trabajo del estudiante que, al final, tiene la capacidad de ofrecer información tanto a enseñantes como a estudiantes sobre los progresos que se están realizando hacia la construcción de significados y explicaciones de carácter científico. Como ejemplos del tipo de tareas curriculares que imaginamos en una aula con cultura de carpeta cabe incluir: Representaciones de problemas, explicaciones y argumentos, y perfiles de preguntas.

\section{Representaciones de problemas}

El proceso de construcción del conocimiento empieza identificando contextos o temas educativos adecuados que definen la orientación a proyectos de las actividades y tareas educativas. Como ejemplo de estos temas cabe citar:

- Determinar la localización de un vertedero de basuras.

- Juzgar la plausibilidad de explicaciones contrapuestas para las causas de los terremotos.

- Diseñar políticas de uso del suelo para el desarrollo de áreas de inundación por parte del municipio.

El tema se selecciona para establecer el objetivo; el siguiente paso estriba en diseñar y desarrollar un trabajo académico que contribuya a la construcción de representaciones de problemas y a la resolución del objetivo. La actividad inicial y las actividades posteriores de representación de problemas podrían pedir a los estudiantes que abordaran las siguientes cuestiones:

- ¿Qué factores se deben tener en cuența en el diseño de un vertedero de basuras?

- ¿Qué información nos haría falta para evaluar la precisión de una explicación de las causas de los terremotos?

- ¿Qué factores se deberían tener en cuenta para comprender los desbordamientos de un río?

Lo que emerge de cada una de estas preguntas tan amplias es el potencial de explorar el conjunto completo de métodos, conceptos científicos, evidencias, principios, leyes, teorías y explicaciones que forman parte de la construcción de una solución significativa para la representación del problema. Por tanto, para los vertederos de basuras, las propiedades del suelo, los registros de pozos y excavaciones, las aguas subterráneas, los mapas topográficos y la estructura del lecho rocoso se convierten en temas viables para ser explorados por los estudiantes. Para las causas de los terremotos, las fuerzas gravitatorias, los modelos del núcleo terrestre, las características geomorfológicas de los continentes y el fondo oceánico se convierten en temas viables, y la localización de los terremotos y los volcanes se convierten en temas de investigación.

Creemos que a partir de estas actividades surgen unas representaciones cada vez más exhaustivas del problema y unas representaciones más significativas de los 
objetivos de las investigaciones científicas. Estas representaciones pueden capturar el desarrollo de la representación de un problema por parte de los estudiantes y ofrecen una rica información a los enseñantes sobre el desarrollo de los estudiantes.

\section{Explicaciones y argumentos}

Un fin u objetivo común que imaginamos para las unidades educativas es la construcción y revisión de argumentos y explicaciones utilizando el marco gradual perfilado anteriormente. Creemos que gran parte de lo que pedimos a los estudiantes debería centrarse en tareas que relacionan la evidencia con la generación de explicaciones científicas o decisiones administrativas. El entorno de aprendizaje de la clase estimulará la articulación y la comunicación (oral y escrita) de explicaciones contrapuestas, y la revisión de explicaciones científicas basadas en evidencias nuevas o en la compatibilidad de la explicación en desarrollo con explicaciones ya establecidas dentro del ámbito de investigación.

Aquí estamos guiados por la estructura epistemológica del ámbito de investigación. A los estudiantes se les enseñarán directrices estratégicas a utilizar en la construcción de explicaciones y argumentos como, por ejemplo, la pauta de argumentos de Toulmin y el Test of Scientific Theories de Giere. Abogamos por la inclusión de las explicaciones de los estudiantes en las carpetas con el fin de evaluar la generación y la evolución de sus explicaciones. También en este caso será rica la información que obtengan los enseñantes sobre el desarrollo conceptual de los estudiantes.

\section{Perfiles de preguntas}

Acompañando a las tareas educativas perfiladas anteriormente se encontrará el planteamiento de preguntas a los estudiantes. Imaginamos el planteamiento de preguntas como otro producto más a incluir en las carpetas. He aquí un ejemplo de cómo los productos existentes en las carpetas mismas pueden convertirse en la base de actividades educativas. Por ejemplo, después de una serie de tareas educativas que se centren en la selección y la evaluación de evidencias utilizadas para juzgar qué explicación es la mejor para la causa de los terremotos, se pediría a los estudiantes que revisaran el trabajo que se encuentra en su fichero y que generaran una serie de preguntas nuevas sobre los terremotos en general, los procedimientos utilizados para recopilar y/o evaluar las evidencias, o la relación entre las evidencias y la construcción de un argumento a favor o en contra de cualquier explicación dada. De nuevo, la génesis de preguntas planteadas por los estudiantes puede arrojar luz sobre el desarrollo cognitivo de estos.

\section{Actividades educativas}

Para conseguir un entorno de aprendizaje en clase que utilice estrategias de enseñanza de cambio conceptual, es necesario abordar diversas relaciones de las estructuras cognitivas en la enseñanza y el aprendizaje. En primer lugar, existe la concepción que tienen los individuos de las declaraciones de conocimiento a aprender. Denominaremos a esta relación «individuo-conocimiento». A continuación se encuentra la relación «estudiante/enseñante-conocimiento». Y por último tenemos la relación «estudiante-conocimiento». Estas tres relaciones reflejan los complejos procesos asociados con la enseñanza y el aprendizaje de la ciencia que se deben abordar mediante actividades educativas.

La investigación sobre la reestructuración del conocimiento en clases de redacción creativa y letras (Gitomer, Grosh y Price, en prensa; Wolf, 1989/1990) indica que los enseñantes pueden modificar estas relaciones tradicionales de una manera radical. En el proyecto Arts $P R O P E L^{4}$, los enseñantes mismos se han visto transformados gradualmente, pasando de ser "dadores de conocimiento" a individuos que 
facilitan una interacción significativa de sus alumnos dentro de un ámbito. Así pues, existe una base práctica para la postura que considera que el desarrollo cognitivo de los niños se representa mejor como una serie de cambios conceptuales.

El problema es que, al aceptar el punto de vista holístico de los cambios conceptuales, los enseñantes desarrollen actividades educativas que se centren en modificar compromisos fundamentales de las estructuras de conocimiento de los estudiantes, tengan éxito en esta empresa, y aun así descubran que se da disonancia o disensión entre los puntos de vista de los estudiantes y los puntos de vista de los expertos en relación a los métodos y los objetivos de la ciencia y, por ende, en relación a la evidencia sobre la cual se sustenta la reestructuración conceptual. Para integrar plenamente las ideas de la epistemología y la ciencia cognitiva en la enseñanza y el aprendizaje de la ciencia, será necesario que los enseñantes de ciencias consideren cómo podrían funcionar estos principios epistemológicos y psicológicos en cada una de las tres relaciones de conocimiento identificadas previamente. El énfasis de la investigación del cambio conceptual recae hoy en la primera relación. Pero una implementación efectiva en el contexto de la clase requerirá que se preste atención a la segunda y tercera relaciones. Los marcos epistemológicos graduales perfilados anteriormente ofrecen el nivel de resolución necesario para guiar la práctica educariva en una cultura de carpeta que pretende reestructurar el conocimiento de los estudiantes.

\section{Criterios de evaluación}

En una cultura de carpeta, la evaluación es mucho más que la administración de exámenes al final de secuencias. Se trata de un proceso en el que se definen criterios, se desarrollan actividades educativas y se construyen mecanismos de evaluación para determinar la calidad del aprendizaje de los estudiantes. Algunos de los criterios que podrían emerger de un modelo gradual del cambio conceptual ya han sido mencionados. De manera similar, hemos hecho referencia a posibles características de actividades educativas. De fundamental importancia es que se dé una alineación coherente entre lo que se valora y las demandas de las actividades de aprendizaje con las que trabajan los estudiantes.

Evidentemente, el trabajo de los estudiantes se debe considerar en términos de firmeza lógica y no en términos absolutos de acierto o error. Como el alcance de las soluciones no se puede prever completamente, es imposible evaluar esta clase de realizaciones sin basarse en las interpretaciones de jueces expertos. Uno de los objetivos de la cultura de la carpeta es ayudar a los enseñantes a convertirse en jueces expertos.

El establecimiento de patrones es fundamental para todo programa de evaluación. Los problemas que se plantean en una clase de ciencias con cultura de carpeta no se prestan a unas respuestas ejemplares ideales que puedan servir para calibrar las respuestas de los estudiantes. El desarrollo de patrones se produce mediante una cuidadosa consideración de la calidad dentro del ámbito y se deriva de la epistemología de la disciplina. Dos argumentos completamente dispares pueden tener una gran calidad, mientras que dos respuestas superficialmente similares pueden variar drásticamente en cuanto a calidad. Por ejemplo, un astuto pensador político puede llegar a las mismas conclusiones que un individuo desinformado en alguna cuestión, pero el argumento del primero puede ser mucho más convincente $\mathrm{o}$, al menos, más coherente.

Los tipos de productos que esperamos de los estudiantes no se someten fácilmente a un análisis lógico formal. Los patrones de lo que constituye una validación y una corroboración aceptable son determinados por una comunidad pertinente. Por tanto, los criterios para una corroboración aceptable pueden ser totalmente distintos para el físico y para el economista. Sin embargo, también en las ciencias naturales se han propuesto esquemas comparables para evaluar la calidad de un argumento (Giere, 1984; 
Root-Bernstein, 1984). En realidad, Duschl (1990) ha afirmado que uno de los objetivos de la enseñanza de la ciencia debería ser enseñar a los estudiantes a evaluar afirmaciones científicas examinando las declaraciones de conocimiento de fondo incluidas en el argumento.

El establecimiento de patrones dentro de un contexto de evaluación requiere más que la identificación de características importantes a considerar. Deben existir unos niveles de rendimiento que sean compartido por los jueces. Aunque la explicación científica es valorada en las exposiciones de proyectos científicos de cuarto curso y en el proceso de revisión de publicaciones periódicas, las expectativas de rendimiento para cada uno de estos contextos es muy diferente. Se han desarrollado modelos de evaluación del rendimiento en varios contextos educativos, incluyendo la puntuación holística de algunos exámenes College Board Advanced Placement (por ejemplo, College Board, 1989), competiciones artísticas, exposiciones científicas, etc.

El establecimiento de patrones debería incluir una comprensión compartida de los criterios utilizados para juzgar el rendimiento. El mismo término puede significar cosas muy distintas para jueces diferentes. Es importante que una discusión para el establecimiento de patrones se dirija a conseguir una comprensión compartida de los conceptos y los aspectos que se van a evaluar. La mejor manera de garantizar esta comprensión es basar la discusión en el trabajo de los estudiantes. Estas discusiones deben tener especificidad. Tanto los estudiantes como los enseñantes deberían apoyar sus afirmaciones sobre el aprendizaje con evidencias específicas. Estudiantes y enseñantes también necesitan desarrollar su capacidad para buscar evidencias de apoyo a la creación de un argumento sobre el aprendizaje de los estudiantes. Las partes implicadas en la evaluación no se deberían limitarse a nociones preconcebidas de que unos tipos específicos de aprendizaje solo son evidentes en determinadas muestras de trabajo.

En esencia, el proceso de evaluación recapitula la empresa científica. La evaluación es una actividad orientada a la comprensión que tiene su base en el trabajo del estudiante y que normalmente se produce en el aula, aunque no está limitada a ella. Los evaluadores (estudiante y enseñante) hacen afirmaciones acerca del aprendizaje del estudiante que deben ser apoyadas por datos y comprobaciones que sean reconocidas como válidos dentro de una comunidad. Quizá, por encima de todo, la evaluación en una cultura de carpeta es, al igual que la ciencia, un proceso de investigación y no una afirmación fija sobre la realidad.

\section{CONCLUSIÓN}

En la enseñanza de la ciencia, la investigación se centra en maneras de provocar cambios conceptuales. Este artículo se ha centrado en el examen de métodos para inducir estos cambios. las consideraciones en torno a lo que es seleccionado por enseñantes/ investigadores para contar como evidencia de que el estudiante pasa del punto de vista $A$ a un punto de vista $B$, se hacen más detalladas y complejas a medida que los estudiantes avanzan en currículo de ciencias K-12. La adopción de una perspectiva evolutiva del crecimiento del conocimiento es lo que nos permite contemplar la aplicación de conceptos de la historia y la filosofía de la ciencia a la enseñanza de la ciencia. Tal como las entienden los filósofos de la ciencia, las actividades de reestructuración del conocimiento comportan la comprobación de declaraciones de conocimiento -el contexto o la lógica de la justificación - y el desarrollo de declaraciones de conocimiento —el contexto o la lógica del descubrimiento-. Tal como las entienden los psicólogos cognitivos, estas actividades comportan ámbitos de conocimiento tanto declarativos como procedimentales. 
Hemos argumentado que una perspectiva evolutiva gradual del cambio conceptual tal como se define filosóficamente, ofrecería citerios muy diferentes para decidir qué enseñar y cómo enseñarlo. La adopción de modelos de enseñanza de cambio conceptual implica facultar al enseñante de una manera que aún hemos de comprender plenamente. Dotar a los enseñantes de unos modelos filosóficos y psicológicos adecuados para la selección y la secuenciación de tareas educativas les ayudaría a describir y prescribir estrategias de aprendizaje efectivas o significativas. La evaluación desempeñaría un papel fundamental para ayudar a los estudiantes a participar en un discurso científicamente importante. Uno de los retos con que se enfrenta la enseñanza de la ciencia es que la naturaleza de la evidencia científica continuará distanciándose de las observaciones basadas en la percepción de los sentidos a medida que continuemos aprendiendo a aprender sobre la naturaleza. Un modelo evolutivo del crecimiento del conocimiento científico ayudaría a estudiantes y enseñantes a organizar los marcos conceptuales de la ciencia y a alcanzar un estado en el que los estudiantes sean capaces de evaluar el grado legítimo de duda asociado a declaraciones de conocimiento científico.

Sin embargo, aunque los modelos de enseñanza y aprendizaje de cambio conceptual hacen recomendaciones específicas sobre actividades y tareas educativas, típicamente operan en base a presuposiciones sobre estudiantes, enseñantes y materias que son difíciles de articular e implementar en clase. Esencialmente, estos modelos ignoran la cuestión de la cultura de la clase que, en nuestra opinión, debe ser abordada. Por ejemplo, los estudiantes, a pesar de la actual enseñanza de la ciencia, son constructores naturales de teorías. Naturalmente, estas teorías suelen ser incompletas (por ejemplo, White y Frederiksen, 1987), incoherentes (por ejemplo, Ranney y Thagard, 1988) y mal orientadas (por ejemplo, Caramazza, McCloskey y Green 1981).

Por tanto, aquí encontramos un ejemplo de cómo la aplicación de la filosofía de la ciencia puede ayudar a definir el papel de la evaluación. Por ejemplo, una importante consecuencia es la recomendación implícita de que la enseñanza que pretenda alterar esquemas conceptuales debe empezar por la evaluación de las ideas que ya posee el estudiante por parte del enseñante. A medida que los estudiantes aprendan a evaluar, se encontrarán en la posición de supervisar de una manera consistente el estado de las cuatro condiciones de Posner et al. Así pues, existe una necesidad de cambiar la responsabilidad de la evaluación para que pase de ser una tarea colocada enteramente en manos del enseñante a ser una tarea compartida por enseñantes y estudiantes.

\section{Notas}

1 Desde la publicación de este importante artículo, Hewson y Thorley (1989) y Strike y Posner (en prensa) han desarrollado con detalle las condiciones necesarias para producir cambios conceptuales en estudiantes. Sin embargo, es el artículo de 1982 el que aparece con más frecuencia en la literatura y, en consecuencia, sigue ocupando una posición de importancia práctica.

2 Se utilizan ejemplares en lugar de paradigmas para evitar el debate de los críticos de Kuhn según los cuales la ciencia no funciona a dos niveles distintos de actividad. Los ejemplares representan los ejemplos compartidos entre científicos y constituyen un aspecto de la matriz disciplinar que, según Kuhn, emplean los científicos en su búsqueda del conocimiento (véase la Figura 1). El mismo Kuhn sustituye ejemplar por paradigma en su nota final (p. 187) a la $2^{\mathrm{a}}$ edición de The Struture of Scientific Revolutions.

3 Aunque el ciclo de aprendizaje de Champagne es similar en cuanto a nombre al modelo didáctico desarrollado por Robert Karplus y ampliado por John Renner, Anthony Lawson y otros, su intención es muy diferente. El ciclo de aprendizaje de Champagne se basa en las teorías psicológicas de Vigotski y Ausubel. El ciclo de aprendizaje de Karplus se basa en las teorías de desarrollo por estadios de Piaget.

4 Arts PROPEL es una colaboración patrocinada por la Rockefeller Foundation en el que intervienen Educational Testing Service, Harvard Project Zero y las Escuelas Públicas de Pittsburgh. Este trabajo se centra en las áreas de las artes plásticas, la música y la redacción creativa en centros de enseñanza media y superior. Para más información sobre Arts PROPEL escribir a Drew Gitomer, ETS, 18-R, Princeton, NJ 08541, E.E.U.U. La Universidad de Pitrsburgh y las Escuelas Públicas de Pittsburgh han iniciado actividades para replicar Arts $P R O P E L$ en las clases de ciencias. 


\section{Referencias}

ANDERSON, C. A., y Smith, E. (1986). Teaching Science. En V. Koehler (Ed.), The educator's bandbook: A research perspective. Nueva York: Longman.

BECHTEL, W. (1990). Scientific evidence: Creating and evaluating experimental instruments and research techniques. En A. Fine, M. Forbes y L. Wessels (Eds.), PSA-1990-Proceedings of the 1990 Biennial Meeting of the Philosophy of Science Association (Vol. 1. pp. 559-572).

Brown, H. I. (1977). Perception, theory and commitment: The new philosophy of science. Chicago: Precedent Publishing Inc.

Brown, J. S.; Collins, A. L., y Duguid, P. (1989). Situated cognition and the culture of learning. Educational Researcher, $18,32-41$

Caramazza, A.; McCloskey, M., y Green, B. (1981). Naive beliefs in "sophisticated" subjects: Misconceptions about trajectories of objects. Cognition, 9, 117-123.

CAREY, S. (1985a). Conceptual change in childbood. Cambridge, MA: Bradford Books.

CAREY, S. (1985b). Are children fundamentally different kinds of thinkers and learners than adults? En S. Chipman et al. (Eds.), Thinking and learning skills (Vol. 2). Hillsdale, NJ: Lawrence Erlbaum Associates.

CAREY, S. (1986). Cognitive science and science education. American Psychologist, 41, 1.123-1.130.

Carey, S.; Evans, R.; Honda, M.; Jay, E., y Unger, C. (1989). "An exriment is when you try it and see if it works": A study of grade 7 students' understanding of the construction of scientific knowledge. International Journal of Science Education, 11, 514-529.

Champagne, A. (1988). A psychological model for science education. Ponencia presentada en la reunión de la American Educational Research Asociation, Nueva Orleans.

$\mathrm{CHI}, \mathrm{M}$. (1990). Conceptual change across ontological categories: Examples from learning and discovery in science. Manuscrito inédito, Universidad de Pittsburgh, LRDC.

COBB, P.; WOOD, T., y YACKEL, E. (1991). Analogies from philosophy and sociology of science for understanding classroom life. Science Education, 75, 23-44.

COGNITION AND TeChNOLOGY GRoup at VANDERBILT (1990). Anchored instruction and its relationship to situated cognition. Education Researcher, $19(5)$.

COLLEGE BOARD (1989). The advanced placement examination in United States history. Nueva York: The College Board.

DuIT, R. (abril 1990). On the role of analogies, similes and metaphors in learning science. Ponencia presentada en el Congreso Anual de la American Educational Research Association, Boston.

DuSCHL, R. (1990). Restructuring science education: The role of theories and their importance. Nueva York: Teachers College Press.

DuSCHL, R.; HAMilton, R., y Grandy, R. (1990). Psychology and epistemology: Match or mismatch when applied to science education? International Journal of Science Education, 12, 220-243.

DusCHL, R., y WRIGHT, E. (1989). A case study of high school teachers' decision making models for planning and teaching science. Journal of Research in Science Teaching, 26, 467-501.

FINLEY, F. (1983). Scientific processes. Journal of Research in Science Teaching, 20, 47-54.

GiBson, B. S. (1985). The convergence of Kuhn and cognitive psychology. New ldeas in Psychology, 3, 211-221.

GIERE, R. (1984). Understanding scientific reasoning (2. ${ }^{a}$ Ed.). Nueva York: Holt, Rinehart and Winston.

GIERE, R. (1988). Explaining science: A cognitive approach. Chicago: University of Chicago Press.

Gitomer, D. H. (1989). Developing a portfolio culture that enables learners. Ponencia presentada en la National Summit Conference on the Arts and Education, Washington, DC.

Gitomer, D.; Grosh, S., y Price, K. (en prensa). Portfolio culture in arts education. Art Education.

Glaser, R. (1984). Education and thinking: The role of knowledge. American Psychologist, 39, 93104.

Gunston, R. F.; White, R. T., y Fensham, P. (1988). Developments in style and purpose of research on the learning of science. Journal of Research in Science Teaching, 25, 513-529.

Hanson, N. (1958). Patterns of Discowery. Cambridge: Cambridge University Press.

Hewson, P., y ThORLey, R. (1989). The conditions of conceptual change in the classroom. International Joumal of Science Education, 11, 541-553.

KitChener, R. F. (1986). Piaget's theory of knowledge: Genetic epistemology and scientific reason. New Haven, CT: Yale University Press.

KITCHENER, R. F. (1987). Genetic epistemology, equilibration and the rationality of scientific change. Studies in the History and Philisophy of Science, 18, 339-366.

KITCHENER, R. F. (en prensa). Piaget's genetic epistemology: Epistemological implications for science education. En R. Duschl y R. Hamilton (Eds.), Philosophy of science, cognitive psychology, and educational theory and practice. Albany: SUNY Press.

Krupa, M.; Selman, R., y JaquetTe, D. (1985). The development of science explanations in children and adolescents: A structural approach. En S. Chipman et al. (Eds.), Thinking and Learning Skills (Vol. 2). Hillsdale, NJ: Lawrence Erlbaum Associates.

KuHN, T. (1962/1970). The structure of scientific revolutions (2. Ed.). Chicago: University of Chicago Press. 
KuHN, D.; AMSHEL, E., y O'LOUGHLIN, M. (1988). The development of scientific thinking skills. Orlando, FL: Academic Press.

LAKATOS, I. (1970). Falsification and the methodology of scientific research programs. En I. Lakatos y A. Musgrave (Eds.), Criticism and the growth of knowledge. Cambridge: Cambridge University Press.

LAMPERT, M. (1990). When the problem is not the question and the solution is not the answer: Mathematical knowing and teaching. A merican Education Research Journal, 27, 29-63.

LAUDAN, L. (1984). Science and values: The aims of science and their role in scientific debate. Berkeley: University of California Press.

LINN, M. (1986). Science. En R. Dillon y R. Sternberg (Eds.), Cognition and instruction. Nueva York: Academic Press.

MinSTRELL, J. (1989). Teaching science for understandding. En L. Resnick y L. Klopfer (Eds.), Toward the thinking curriculum: Current cognitive research. 1989 Yearbook of the Associacion for Supervision and Curriculum Development. Reston, VA: ASCD.

Mitman, A. L.; Mergendoller, J. R.; Marchman, V. A., y Packer, M. J. (1987). Instruction addressing the components of scientific literacy and its relation to student outcomes. American Educational Research Journal, 24, 611-633.

NerSESSIAN, N. (1989). Conceptual change in science and in science education. Synthese, 80, 163 183.

NovaK, J. (1977). A theory of education. Ithaca, NY: Cornell University Press.

Novak, J., y GowIN, R. (1984). Learning how to learn. Cambridge: Cambridge University Press.

Nussbaum, J. (1983). Classroom conceptual change: The lesson to be learned from the history of science. En H. Helm y J. Novak (Eds.), Proceedings of the International Seminar on Misconceptions in Science and Mathematics. Ithaca, NY: Department of Education, Cornell University.

OSBORNe, R., y FreYBerg, P. (Eds.) (1985). Learning in science: The implications of children science. Londres: Heinemann Press.

OSBORNE, R., y WiTTROCK, M. (1983). Learning science: A generative process. Science Education, 67, 489-508.

PIAGeT, J. (1970). Six psychological studies. Nueva York: Vintage Books.

Posner, G.; Strike, K.; Hewson, P., y Gertzog, W. (1982). Accommodation of a scientific conception: Toward a theory of conceptual change. Sciencce Education, 66, 211-227.

RANNEY, M., y THAGaRD, P. (1988). Explanatory coberence and belief revision in naive physicos. (Cognitive Science Laboratory Report No. 31). Princeton, NJ: Princeton University.

RESNICK, L. (1983). Mathematics and science learning: A new conception. Science, 220, 477-478.

RESNICK, L., y KLOPFER, L. (Eds.) (1989). Toward the thinking curriculum: Current cognitive research 1989 ASCD Yearbook. Reston, VA: ASCD.

RISSLAND, E. (1985). The structure of knowledge in complex domains. En S. Chipman et al. (Eds.), Thinking and learning skills (Vol. 2). Hillsdale, NJ: Lawrence Erlbaum Associates.

RoOt-Bernstein, R. (1984). On defining scientific theory: Creationism considered. En A. Montague (Ed.), Science and crestionism (pp, 64-94). Nueva York: Oxford University Press.

RoTH, K. (1989). Science education: It's not enough to "Do" or "Relate". American Educator, Invierno, 16-22 y 46-48.

Roth, K. J.; ANDERSON, C. W., y SMITH, E. L. (1987). Curriculum materials, teacher talk and student learning: Case studies in fifth grade science teaching. Joumal of Curriculum Studies, 19, 527 548.

SCHAUBLE, L., y KLOPFER, L. (1990). Children's conceptualization of scientific inquiry: Explorations in bydrostatics and bydrodynamics. Ponencia presentada en la Annual Meering of the American Educational Research Association, Boston.

SHAPERE, D. (1984). Reason and the search for knowledge: Investigation in the philosopby of science. Dordrecht, Holanda: Reidel Press.

STrike, K., y POSNER, G. (en prensa). A revisionist theory of conceptual change. En R. Duschl y R. Hamilton (Eds.), Philosopby of science, cognitive psychology, and educational theory and practice. Albany: SUNY Press.

SUPPE, F. (Ed.) (1977). The structure of scientific theories (2. Ed.). Urbana-Champagne, IL: University of Illinois Press.

TAKeUChI, H.; Uyeda, S., y Kanamori, H. (1967). Debate about the earth: Approach to geophysics through analysis of continental drift. San Francisco: Freeman, Cooper \& Co.

Thagard, P. (1990). The conceptual structure of the chemical revolution. Philosophy of Science, 57, 183-209.

Tobin, K., y Gallagher, J. (1987). What happens in high school science classrooms? Journal of Curriculum Studies, 19, 549-560.

TREAGUST, D., y FetherstonHAUGH, T. (abril 1990). Student's understanding of light and its properties following a teaching strategy to engender conceptual change. Ponencia presentada en la Annual Meering of the American Educarional Research Association, Boston.

VOSNIADOU, S., y BREWER, W. F. (1987). Theories of knowledge restructuring in development. Review of Educational Research, 57, 51-67.

WEST, L., y PINES, A. (Eds.) (1985). Cognitive structure and conceptual change. Nueva York: Academic Press. 
WHITE, B., y FREDERIKSEN, J. (1987). Progressions of qualitative models as a foundation for intelligent learning environments (BBN Report No. 6277). Cambridge, MA.

WhitT, L. A. (1989). Conceptual dimensions of theory appraisal. Studies in History and Pbilosophy of Science, 19, 517-529.

WHITT, L. (1990). Theory Pursuit: Between discovery and acceptance. En A. Fine, M. Forbes y L. Wessels (Eds.), PSA 1990 - Proceedings of the 1990 Bieenial Meeting of the Pbilosopby of Science Association (Vol. 1, pp. 467-484).

WOLF, D. P. (1989/90). Portfolio assessment: Sampling student work. Educational Leadersbip, 47, 3539.

ZEITSMAN, A., y ClEMENT, J. (abril 1990). Using anchoring conceptions and analogies to teach about levers. Ponencia presentada en la Annual Meeting of the American Educational Research Association, Boston.

\section{Perspectivas epistemológicas sobre el cambio conceptual: Implicaciones para la práctica educativa Richard A. Duschl CL\&E; 1995, 25, pp. 107-125}

Resumen: Los marcos de referencia que persiguen comprender cómo se produce la reestructuración del conocimiento y cómo construir un entorno de aprendizaje que facilice esta reestructuración, plantean importantes interrogantes filosóficos, psicológicos y pedagógicos y cuestiones sobre cómo se produce el cambio conceptual y qué caracrerísticas del crecimiento del conocimiento deberían formar parte de los currículos y de los entornos de aprendizaje. En el hecho de destacar el cómo, se encuentra implícito un cambio en la perspectiva de la enseñanza de la ciencia, que va desde abrazar 'los modos de conocimiento de los científicos' como objetivo dominante, a favorecer 'el posicionamiento del estudiante para el próximo paso'. Este cambio de perspectiva y de enfoque representa un desvío radical y complejo respecto a la práctica común. En este artículo se presenta un modelo gradual del carácter y el mecanismo de la reestructuración y, a continuación, se describe un modelo de prácrica educativa diseñado para facilitar esta forma de reestructuración. Nosotros sostenemos que una perspectiva evolutiva y gradual del cambio conceptual ofrecería unos criterios totalmente diferentes para decidir qué enseñar y cómo enseñar. La adopción de modelos de enseñanza basados en el cambio conceptual implica facultar al enseñante de una manera que aún hemos de comprender plenamente. Dotar a los enseñantes de modelos filosóficos y psicológicos adecuados para la selección y la organización secuencial de tareas educativas les ayudaría a describir y prescribir estrategias de aprendizaje efectivas o significativas. En este modelo educativo ocupa un lugar central un punto de vista ampliado e integrado de la evaluación y la enseñanza que nosotros denominamos cultura de la carpeta. La característica esencial de esta cultura es que crea oportunidades para que enseñantes y estudiantes puedan confrontar y desarrollar su comprensión científica, y para equipar a los estudiantes con las herramientas necesarias para aumentar su responsabilidad por su propia reestructuración y para que evalúen por sí mismos cuáles pueden ser los siguientes pasos.

Artículo original; «Epistemological Perspectives on Conceptual Change: Implications for Educational Practice». Journal of Research in science Teaching, vol. 28, $N^{\circ} 9$, pp. 839-858 (1991). Reproducido con autorización de John Wiley \& Sons, Inc. Traducción de Genis Sánchez Barberán.

Dirección de los autores: Richard A. Duschl Department of Instruction and Learning and the Center for Philosophy of Science, University of Pittsburgh, Pennsylvania 15260 Drew H. Gitomer Educational Testing Service, Princeton, New Jersey 08541

C PERMISOS PARA CITAR O REPRODUCIR EN OTRAS FUENTES: Se pueden citar libremente hasta 500 palabras. Para reproducir una porción de texto mayor, figuras o ilustraciones, se deberá pedir permiso por escrito a la revista, especificando el uso al que se destina el texto. En todos los casos, se deberá citar el copyright de $C L \& E$. En el caso de artículos o textos que hayan sido a su vez reproducidos en $C L \& E$ los interesados deberán dirigirse tanto a los detentadores del copyright original como a $C L \& E$, en el caso de que se quiera hacer uso de la traducción. FO'TOCOPIAS: Para todo lo relacionado con el uso mediante fotocopia del material de esta revista, deberán dirigirse a: CEDRO, C/ José Marañón, 10, $3 .^{\circ}$ Izda. Tel. 5941575 . Fax 4453567 